\title{
New EuroGentest/ESHG guidelines and a new clinical utility gene card format for NGS-based testing
}

\author{
European Journal of Human Genetics (2016) 24, 1; doi:10.1038/ejhg.2015.229; published online 28 October 2015
}

$\mathrm{T}$

This issue of the European Journal of Human Genetics presents, on 1 behalf of EuroGentest and the European Society of Human Genetics (ESHG), guidelines for the evaluation and validation of next-generation sequencing (NGS) applications for the diagnosis of genetic disorders. ${ }^{1}$ They address a wide spectrum of points to consider, including a definition of the diagnostic utility of an NGS test, a ranking of NGS assays on the basis of essential vs optional genes they might include, standardization of quality parameters, policies for dealing with the additional features that are intrinsic to NGS testing, such as secondary and unsolicited findings, and distinctions to be made depending on the research $v s$ diagnostic setting. The guidelines stress the necessity for frequent updating in this rapidly evolving field.

These guidelines propose that groups of clinical and laboratory experts should define 'core gene lists' that must be analysed in any particular diagnostic or research approach $v s$ additional genes that might be added, but cannot be considered as mandatory, mainly because of their low diagnostic yield. Selecting these core genes is not trivial, because even the definition of a core gene is still under debate. Evidently, the frequency of mutations in the gene in relation to the phenotype is an important parameter. But it is not easy to define the threshold: can a gene that explains less than $1 \%$ of the cases be a core gene? Probably not, but practitioners like to have the largest possible yield when ordering a test. At the same time, NGS is appealing as one can easily increase the number of genes in a diagnostic panel. Shall a core gene be completely sequenced, and shall a core gene panel be a 'type A' test, as defined in the diagnostic NGS guidelines? ${ }^{1}$ Yes if it is about warranting maximal sensitivity, no if it is about filling up-using Sanger sequencing for instancegaps in the sequence in exons (or functional domains of the protein) in which no one has ever detected mutations, even in large cohorts. Thus, it is not decided yet whether we are developing 'core gene lists' or 'core exon lists'. But irrespective of that discussion, it is time for people to get together and publish core gene/exon lists, and harmonize the diagnostic offer for the sake of the patients.

As a prototype for such a group consensus paper this issue also publishes the first NGS-adapted Clinical Utility Gene Card for Hereditary Thoracic Aortic Aneurysm and Dissection (TAAD). ${ }^{2}$ We hope that the scientific and clinical community will find it useful to find a specific example for one of the recommendations of the EuroGentest/ESHG NGS guideline, and that it might inspire the readership for further consensus papers of this kind, and for reading and applying the diagnostic NGS guidelines.

\section{CONFLICT OF INTEREST}

The authors declare no conflict of interest.

Gert Matthijs $^{1}$, Anna Dierking ${ }^{2}$ and Jörg Schmidtke ${ }^{2}$

${ }^{1}$ Department of Human Genetics, Centre for Human Genetics, KU Leuven, Leuven, Belgium;

${ }^{2}$ Institute of Human Genetics, Hannover Medical School, Hannover,

Germany

E-mail: gert.matthijs@uzleuven.be

1 Matthijs G, Souche E, Alders M et al: Guidelines for diagnostic next generation sequencing. Eur J Hum Genet 2016; 24: 2-5.

2 Arslan-Kirchner M, Arbustini E, Boileau C et al: Clinical utility gene card for: Hereditary Thoracic Aortic Aneurysm and Dissection (TAAD) Including Next Generation Sequencing Based Approaches. Eur J Hum Genet 2016; 24: 146. 